\title{
L'urbanisme transitoire à Montréal : entre innovation et préservation
}

\author{
Taiika Baillargeon ${ }^{a}$, Jérémy Diaz ${ }^{b}$
}

RÉSUMÉ. Longtemps associées à l'activisme et à la transgression, les initiatives d'occupation temporaire des sites vacants sont désormais utilisées par les municipalités dans leur stratégie de réaménagement ainsi qu'envisagées comme vecteur de l'économie créative et comme moteur d'innovation (Bishop et Williams, 2012; Colomb, 2012; Ginez, 2018; Mould, 2014; Pinard et Vivant, 2017). Plusieurs chercheurs envisagent d'ailleurs qu'elles deviennent monnaie courante, s'intégrant à la planification traditionnelle (Bishop et Williams, 2012; Zielh, Oßwald, Hasemann et Schnier, 2012). Dans ce contexte, on parle de plus en plus d'urbanisme transitoire : une pratique qui est autorisée, planifiée et perçue comme une étape de préfiguration pour des projets de requalification pérennes. Qu'est-ce que l'urbanisme transitoire? Comment se distingue-t-il des autres formes d'urbanisme temporaire et comment se déploie-t-il dans le contexte montréalais? Autant de questions auxquelles nous tenterons de répondre dans cet article.

\begin{abstract}
$\boldsymbol{A B S T R A C T}$. Temporary occupation of vacant sites was long associated to activism and encroachment. V acant sites are now increasingly used by municipalities in their development strategies, and considered as vectors of creative economy and the driving force of innovation (Bishop \& Williams, 2012; Colomb, 2012; Ginez, 2018; Mould, 2014; Pinard \& Vivant, 2017). Many authors even believe they will become common practice and will integrate traditional planning (Bishop \& Lewis, 2012; Zielh, Oßwald, Hasemann, \& Schnier, 2012). In this context, we are increasingly talking about "transitory town planning", a planned and authorized practice that is perceived as a prefiguration step to lasting requalification projects. What is transitory urbanism? How does it stand out from other temporary town planning practices and how does it become implemented in Montreal? These are a few questions we will try to answer in this article.
\end{abstract}

\section{Introduction}

Depuis deux décennies, les interventions urbaines dites temporaires se sont accélérées de manière phénoménale (Bishop et Lewis, 2012; Douglas, 2013; Hou, 2010; Iveson, 2013; Lydon et Garcia, 2015). Sur les terrains vagues et dans les bâtiments vacants des centres-villes, des initiatives d'occupation et d'aménagement de plus en plus variées, de différentes envergures et de différentes temporalités apparaissent et disparaissent : des commerces $p \circ p$ - $и$, des bars, des espaces de travail partagés, des espaces festivaliers ou de simples installations ludiques et invitantes. Le phénomène est tel qu'on parle aujourd'hui d'une « ville temporaire » et $p \circ p-u p$ : une ville qui se développerait en parallèle de la planification traditionnelle, conçue pour une permanence qui ne semble plus convenir à notre époque d'ambivalence, d'incertitude et de rapidité (Beekmans et
De Boers, 2014; Bishop et Williams, 2012). Si ces initiatives ont deux grands dénominateurs communs - l'occupation des espaces vacants et une temporalité limitée -, elles se distinguent de différentes manières, marquées par des contextes et par des motivations qui évoluent.

Historiquement, les occupations temporaires ont largement été engendrées de manière marginale et informelle par des individus ou par des groupes citoyens. La littérature sur le sujet nous ramène notamment aux mouvements d'engagement civique du XIXe siècle et aux squats des décennies 1960, 1970 et 1980, mais on mentionne également les installations éphémères de la ville événementielle des années 1990 (Chaudoir, 2007; IAU, 2018a; Talen, 2015).

\footnotetext{
a Professeure associée, département de géographie, Université du Québec à Montréal

b Doctorant, département d'études urbaines et touristiques, Université du Québec à Montréal
} 
Depuis le début des années 2000, les initiatives temporaires semblent toutefois de moins en moins marginalisées. Non seulement constatons-nous un nombre croissant d'initiatives temporaires, mais ces dernières sont de plus en plus autorisées et planifiées, bien souvent organisées avec l'appui de paliers institutionnels. Plusieurs chercheurs avancent même que ces pratiques pourraient devenir monnaie courante et s'intégrer à la planification traditionnelle (Bishop et Lewis, 2012; Zielh et collab., 2012). Plusieurs villes européennes travaillent d'ailleurs dans ce sens. Par exemple, avec le New London Plan de 2018, la Ville de Londres propose une politique d'usage temporaire. Plus récemment, la Ville de Paris a signé une charte pour le développement de l'occupation transitoire avec environ 15 partenaires publics et privés.

Dans les milieux francophones, ces initiatives autorisées et planifiées de manière officielle sont de plus en plus identifiées comme de "l'urbanisme transitoire ». Cette pratique d'aménagement, qui permet l'occupation de sites temporaire à court ou à moyen terme, a la particularité de viser la permanence des projets (Pradel, 2018). L'urbanisme transitoire est ainsi considéré comme une étape préalable à la mise en place d'un projet pérenne; il sert à alimenter et à préfigurer des usages durables dans un espace donné.

Pour mieux comprendre cette pratique innovante et comment elle se développe sur le territoire montréalais, cet article propose d'abord d'en préciser la nature, d'en retracer l'histoire et d'en mettre en lumière les spécificités. Il sera ensuite question de présenter le cas montréalais et les jalons qui mènent au transitoire à Montréal. En conclusion, nous aborderons les limites de la pratique, soulignant notamment la professionnalisation et la popularisation de l'occupation temporaire.

\section{1. Éléments théoriques sur l'urbanisme transitoire}

Parallèlement aux initiatives d'occupation temporaire qui se sont multipliées au cours des deux dernières décennies, la recherche sur le sujet s'est également largement développée au cours des 10 dernières années (Andres, 2013). Dans ce contexte, les termes "occupation temporaire» et "urbanisme temporaire » sont les plus couramment utilisés pour décrire ces initiatives de court ou moyen terme qui prennent place dans des sites vacants urbains.

Selon l'Institut d'aménagement et d'urbanisme de la région Île-de-France, l'urbanisme temporaire se réfère à «toutes les initiatives qui visent, sur des terrains ou bâtiments inoccupés, à réactiver la vie locale de façon provisoire, lorsque l'usage du site n'est pas encore décidé, ou le temps qu'un projet se réalise » (IAU, 2018b, p. 20). Ces pratiques variées partagent deux dénominateurs communs: des espaces vacants (intérieurs ou extérieurs) et une temporalité limitée.

Selon Andres (2013), la littérature approche le domaine sous deux angles bien distincts: les espaces culturels et les squats (Groth et Corijn, 2005; Chatterton et Hollands, 2003; Pruijt, 2003) et les activités économiques et culturelles dans les zones abandonnées (Haydn et Temel, 2006; Oswalt, 2005; Overmeyer, 2007; Urban Unlimited, 2004). Dans cette section, nous aborderons ces deux angles. Nous définirons la pratique en présentant ses caractéristiques principales, puis nous en déplierons les diverses facettes, sous un angle plus historique.

\subsection{L'urbanisme temporaire : la vacance et le provisoire}

La vacance est le premier élément au cœur de ces initiatives. Symptomatique du développement urbain et du changement, la vacance est un problème qui persiste dans les grandes villes depuis longtemps, une réalité intrinsèque à la ville moderne. Or, elle devient problématique en temps de crise, lorsque les espaces et bâtiments vacants se multiplient et que le marché immobilier est au ralenti. Le développement industriel et la désindustrialisation, grandes époques de production capitaliste, ont notamment été d'importants moteurs de vacance. Les crises économiques, les guerres et les catastrophes naturelles sont d'autres causes de vacance qui pèsent lourd sur la gestion et la perception des villes ainsi que sur les paysages urbains. Il existe également quelques raisons contextuelles, par exemple l'état et la symbolique des lieux, l'évolution naturelle des villes et les changements de nos modes de vie, qui expliquent la vacance.

Dans nos villes contemporaines, la vacance est très généralement perçue comme un problème. D’une part, les bâtiments laissés dans un abandon complet ou partiel sont très généralement affectés dans leur 
structure : avec le temps, la vacance fragilise le bâti, puis pose d'abord et avant tout un problème de sécurité. On sait depuis longtemps, par exemple, que l'abandon pose un important risque d'incendie, dangereux pour le bâtiment et pour le voisinage (Mallach, 2006; Schachterle, Bishai, Shields, Stepnitz et Gielen, 2012). À une échelle à la fois environnementale et économique, l'abandon d'un bâti constitue également une perte de ressources (Mallach, 2006; Garvin, Branas, Keddem, Sellman et Cannuscio, 2013). À une échelle patrimoniale, un bâtiment historique en déclin est de plus en plus difficile à préserver et sa valeur architecturale peut être grandement affectée.

La vacance peut également avoir un impact sur le sens du lieu. En effet, à partir du moment où le bâtiment vacant pose problème pour le voisinage, son image s'en ressent également. D'un point de vue esthétique, ces lieux jurent avec notre perception du beau, qu'on associe le plus souvent au paysage organisé et continu, et, d'un angle plus fonctionnel, l'absence et l'insignifiance contribuent au sentiment d'insécurité (Korosec-Serfaty, 1991; Lévesque, 1999; Veschambre, 2008; Vidler, 1992). Il est d'ailleurs intéressant de noter qu'en anglais, on parle souvent de pollution visuelle (eyesore) ou de délabrement (blight) lorsqu'on parle de vacance.

Ainsi, la vacance est qualifiée d'illégitime, de dangereuse, de louche ou de sinistre, et cette désignation influence aussi notre perception. Il est vrai qu'il s'agit d'espaces qui sortent des réseaux traditionnels de la ville, et leur situation comme leur état incitent aux activités anonymes et illicites. On peut notamment penser aux occupations illégales qualifiées de sans-abrisme ou aux opérations de ferrailleurs. On peut également penser à des pratiques à la fois perçues comme criminelles et/ou artistiques (p. ex., le graffiti et l'exploration urbaine).

Ces différents usages participent parfois à la dégradation du bâti, mais il arrive également qu'ils incitent à la préservation par l'urgence ou par le moyen de pratiques artistiques. Comme le suggèrent plusieurs auteurs, les sites vacants ont aussi un potentiel de développement incomparable. Ils sont en quelque sorte le lieu de toutes les potentialités (Andres, 2011; Beaudet, 1997; Nefs, 2006). Doron (2007) fait d'ailleurs le lien entre ces espaces interstitiels et la chôra (ou khôra) de la Grèce antique. En effet, à l'époque des villes-forteresses, la chôra était l'espace qu'on retrouvait au-delà des murs de la ville; un territoire mi-rural, mi-nature qui était généralement militarisé parce qu'il s'agissait aussi d'un entre-deux entre la ville et les voisins rivaux. Selon Platon, nous explique Doron, cette chôra sans identité propre ou permanente était considérée comme un espace de création : «un emplacement où les choses étaient formées » (Doron, 2006, p. 18 , trad. libre).

Tel que le sous-entend la terminologie, l'urbanisme temporaire est déterminé par un espace, mais aussi par une temporalité provisoire. Encore une fois, il existe toutefois quelques variations: un projet d'urbanisme temporaire peut durer le temps d'une soirée, d'un trimestre ou de quelques années. Il peut aussi être périodique ou cyclique. Il n'est d'ailleurs pas surprenant que le terme meanwhile uses (usages en attendant) soit couramment utilisé chez les Anglo-Saxons, alors qu'en allemand on utilise plutôt zwischennutzungen (entre deux usages). Ces deux termes témoignent bien du caractère temporaire des initiatives en question, mais, en même temps, ils soulignent le caractère opportuniste de ces pratiques: on construit ici entre les mailles spatio-temporelles du développement urbain traditionnel et du marché immobilier spéculatif (Tonkiss, 2013). À une autre échelle, même si les initiatives d'occupation temporaire sont de courte ou de moyenne durée, elles reflètent aussi une volonté de participer à changer la ville et la façon de la construire à long terme. Pour Pradel (2018), l'urbanisme temporaire est d'ailleurs beaucoup plus qu'un usage provisoire des espaces vacants. C'est un processus de revalorisation des espaces qui pose problème :

L'action d'organiser et d'aménager temporairement ou cycliquement des espaces à enjeux, publics ou privés, ouverts ou bâtis, occupés ou inoccupés, afin d'en stimuler les usages, d'y amplifier les échanges et d'y générer des pratiques à court terme dans une perspective de valorisation symbolique, (ré)investissement social et transformation spatiale à long terme. (Pradel, 2018)

Cette définition est encore très englobante, tenant compte de diverses formes de pratiques et de divers territoires, mais elle nous ramène surtout à la question des motivations: Pourquoi fait-on de l'urbanisme temporaire? 


\subsection{Le squat et l'urbanisme tactique : l'engagement citoyen et le droit à la ville}

Selon la littérature, l'occupation temporaire de sites vacants existe depuis longtemps et participe, de diverses manières, au renouvellement des villes (Andres, 2013; Bossetti et Colthorpe, 2018; Ginez, 2018; Talen, 2015). C'est un phénomène qu'on observe notamment au lendemain de grandes crises. Pensons par exemple aux occupations qui suivent la Première ou la Seconde Guerre mondiale en Europe (Ginez, 2018; Vasudevan, 2017). En Angleterre, en France et aux Pays-Bas, l'occupation des bâtiments vacants tient déjà de la résistance; il s'agit d'une façon de lutter pour l'accès au logement (Van der Steen, Katzeff et Van Hoogenhuijze, 2014; Vasudevan, 2017).

Les auteurs qui s'intéressent à l'histoire de l'urbanisme temporaire tel qu'on le connait aujourd'hui nous ramènent toutefois le plus souvent aux mouvements de squat qui se développent en Europe et aux États-Unis à partir de 1968 (Adisson, 2017; Ambrosino et Andres, 2008; Dubeaux, 2017; Jégou et Bonneau, 2016; Oswalt, Overmeyer et Misselwitz, 2013; Tonkiss, 2013; Pinard et Vivant, 2017). Le squat est un mot d'origine anglaise qui définit l'action illégale d'occuper un édifice inhabité ou de s'installer sur un terrain vacant. À partir de 1968, au lendemain de mai 68 en France et au cœur de la guerre du Vietnam, l'Europe et les États-Unis deviennent le berceau de mobilisations urbaines menées par une jeunesse radicale et libertaire (Van der Steen et collab., 2014).

À l'échelle de la ville, on remet en question la planification fonctionnaliste et on réclame l'intervention d'acteurs non institutionnels. Le philosophe français Henri Lefebvre (1970) publie La révolution urbaine, tandis que la Canado-Américaine Jane Jacobs milite pour la préservation des quartiers et l'implication citoyenne. "L'appropriation de l'espace endosse un rôle d'expression politique » (Ginez, 2018, p. 31). À cette époque, le squat est principalement un outil de lutte citoyenne pour le droit au logement.

Cette pratique d'occupation est initialement illégale, mais elle n'est pas toujours contestée par la population locale. C'est notamment le cas lorsque les revendications des squatteurs se rattachent à une lutte pour la conservation du patrimoine bâti. Comme si la lutte pour le patrimoine donnait un certain sérieux à des pratiques marginales et informelles. Le cas des squats à Berlin-Ouest au début des années 1970 en est un bon exemple. Si le mouvement est accepté et même appuyé par une certaine partie de la population locale, c'est qu'il revendique la préservation d'un bâti caractéristique des quartiers ouvriers de Berlin-Ouest à une époque où on rase tout, malgré une importante crise du logement. Comme le font remarquer plusieurs auteurs, il faut d'ailleurs rappeler que les pratiques d'occupation temporaire tanguent depuis longtemps entre la sphère institutionnelle et la légalité :

Les occupations sont tour à tour illicites, tolérées ou contrôlées (notamment par le conventionnement des squats), ou bien soutenues voire imposées (cadres juridiques sécurisés, mesures d'incitation ou d'accompagnement financier, matériel ou symbolique, baux flexibles, bas loyers, etc.) par les élites urbaines politiques, économiques ou culturelles. (Ginez, 2018, p. 19)

Dans les décennies 1980 et 1990, les centres-villes des grandes villes industrielles connaissent d'importants problèmes de vacance. Les mouvements de squat se multiplient et se radicalisent davantage, à une époque de transition économique et de récession. Les occupations sont d'abord lancées par des anarchistes, puis des altermondialistes; les discours évoluent, mais restent dans un esprit de revendication et d'hostilité envers le système établi.

À cette époque, les squats sont aussi des hauts lieux de la contre-culture; ils sont notamment appropriés par la culture punk, puis par la scène électro. Les édifices vacants se font d'excellents lieux de concerts informels et de raves (Van der Steen et collab., 2014). Les mouvements de squat continuent aujourd'hui d'évoluer au même rythme que les villes; la lutte pour l'accès au logement reste fondamentale, mais les discours qui entourent les pratiques se rattachent à des luttes plus contemporaines: oppression des genres, précarité des migrants, etc.

Tout comme les mouvements de squat, l'urbanisme tactique prend racine dans l'engagement citoyen et tangue avec la légalité. Comme le mentionne Plateau urbain (2018), cette forme d'urbanisme est fortement rattachée au mouvement d'urbanisme temporaire. On 
pourrait même avancer qu'elle en est le premier jalon. L'urbanisme tactique ou do it yourself (DIY) fait référence à différentes pratiques et interventions de très petite échelle qui sont entreprises par des résidents (Iveson, 2013; Talen, 2015). L'idée derrière cette pratique est qu'il est possible de participer à transformer son quotidien sans passer par un vecteur politique ou économique; de transformer la ville sans passer par l'urbanisme formel. Les mobilisations éclairs (flash mobs), les guérillas jardinières (guerrilla gardening), les arts de la rue et toute autre forme d'installations informelles peuvent être d'excellents exemples d'urbanisme DIY. Ces pratiques sont très généralement situées dans les terrains vagues, mais, comme le souligne le collectif Plateau urbain (2018), «l'entrée ne se fait pas tant par le temporaire que par l'ouverture au public et la volonté de faire participer les citoyens » (p. 9).

Selon Lydon et Garcia (2015), l'urbanisme tactique a toujours existé :

The city- and place-making process we now call tactical urbanism is not [new]. Indeed the development of human settlements has always included, if not required, incremental and selfdirected action aimed towards increasing social capital, commercial opportunity and urban livability. In many developing countries, this remains the only way forward. (p. 2)

Toujours selon le collectif, la vague d'urbanisme tactique qu'on connait actuellement serait toutefois attribuée à trois éléments distincts : 1) la récession; 2) les changements démographiques; et 3) l'Internet comme outil de développement d'une économie de partage (Lydon et Garcia, 2015). Comme c'est le cas pour les mouvements de squat, les pratiques d'urbanisme tactique telles qu'on les connait aujourd'hui prennent toutefois leurs racines dans les mouvements d'occupation citoyenne de la fin des années 1960. Or, si les squats revendiquent alors le droit au logement, l'urbanisme tactique témoigne d'une volonté de participer à faire la ville. Ces pratiques servent ainsi à revendiquer des changements dans la planification traditionnelle: la mise en place et/ou l'embellissement des espaces publics, la mise en place de pistes cyclables ou de passages piétonniers, etc. En ce sens, bien que les initiatives d'urbanisme tactique soient très souvent ludiques, elles se distinguent de l'urbanisme éphémère qui, selon Pradel (2018), reste fondamentalement à vocation événementielle et festive, et répond essentiellement d'une «dynamique de réversibilité fonctionnelle ».

Il est intéressant de noter que, dans une visée d'engagement citoyen, ces pratiques d'urbanisme tactique se rattachent aussi à la méthode DIY. En effet, si les initiatives d'urbanisme tactique sont très variées, elles restent généralement accessibles à tous, souvent instaurées avec peu de coûts et construites avec des matériaux recyclés. Elles sont aussi bien souvent organisées collectivement de manière à assurer un partage de l'espace et une mutualisation des ressources et des responsabilités. Lydon et Garcia (2015) décrivent ainsi ces initiatives comme ayant peu de risques, mais beaucoup de potentiel (low-risk, high-reward) pour les quartiers et les villes.

C'est d'ailleurs pour ces mêmes raisons que les acteurs issus de la planification traditionnelle, autant du côté public que privé, s'intéressent de plus en plus à ces pratiques. Aussi ludiques et sympathiques soient-ils, les aménagements d'urbanisme tactique sont aussi critiqués, reconnus comme moteur de gentrification, génèrent souvent des impacts néfastes à long terme, en plus d'encourager la déresponsabilisation de l'État (Mould, 2014). On remet en question également l'approche très sporadique de cet urbanisme, qui fonctionne généralement par projet, sur un territoire bien spécifique, sans porter un regard plus large sur le développement urbain (Mould, 2014). Il faut dire qu'une fois les quartiers réanimés, il n'est pas rare que les lieux initialement vacants soient réinvestis par le marché traditionnel, sans que les communautés qui ont participé à réanimer les lieux puissent en profiter (Andres, 2011; Colomb, 2012).

Le droit au logement et la participation citoyenne à la fabrication de la ville sont tous deux au cœur du concept de «droit à la ville», qui est fondamental aux initiatives d'occupation et d'urbanisme temporaires. En effet, les différents mouvements qui s'apparentent à cette « ville temporaire » s'inscrivent en continuité avec ce concept réfléchi par le philosophe Henri Lefebvre dans les années 1960, qui affirme que le droit à la ville est une :

Forme supérieure des droits : droit à la liberté, à l'individualisation dans la socialisation. Le droit à l'œuvre (à l'activité participante) et le droit à l'appropriation (bien distinct du droit à la propriété) impliquent le droit à la vie urbaine. (Lefebvre,1968, p. 140) 
Comme le rappelle Harvey (2012), il s'agit de revendiquer l'accès au logement, aux espaces publics, au centre-ville, mais il s'agit aussi de revendiquer le droit à changer la ville collectivement. Ce concept s'inscrit dans le contexte d'une urbanisation massive que reconnait déjà Henri Lefebvre et qui s'est accentué dans les dernières années. En effet, dans La révolution urbaine, Lefebvre (1970) prophétisait « l'urbanisation complète de la société » (p. 9) et annonçait ainsi un passage vers une "zone critique ", qu'il présentait comme un "second balancement [après le passage de la ville commerciale à la ville industrielle], une seconde inversion de sens et de situation [où] l'industrialisation, puissance dominante et contraignante, se change en réalité dominée au cours d'une crise profonde, au prix d'une énorme confusion, dans laquelle le passé et le possible, le meilleur et le pire s'enchevêtrent» (p. 26).

Cette situation pousse alors le philosophe à réfléchir à une tierce voie pour libérer le citoyen de la dominance capitaliste. Pour Lefebvre, la révolution urbaine doit se faire à partir d'une participation citoyenne à la fabrication de la ville. Dans La production de l'espace (Lefebvre, 1974), la pratique de l'espace devient d'ailleurs un geste politique qui génère le changement et qui permet la révolution urbaine (ou le mouvement contre la fixité).

Aujourd'hui, à une époque de conscientisation environnementale, la question du droit à la ville devient également une question de durabilité : l'héritage bâti doit être réutilisé de manière à profiter aux générations futures. La question de la durabilité semble d'ailleurs prendre de plus en plus d'importance dans les milieux du temporaire.

\subsection{L'urbanisme transitoire : une occupation autorisée, mais durable}

L'urbanisme temporaire prend donc racine dans les mouvements de lutte et d'engagement citoyens qui se développent à partir de la fin des années 1960. Aujourd'hui, non seulement ces initiatives se multiplient, mais on remarque également qu'elles sont de plus en plus envisagées comme vecteur de l'économie créative, circulaire et sociale, et utilisées par les municipalités dans leur stratégie de réaménagement (Bishop et Lewis, 2012; Colomb, 2012; Mould, 2014) :

Many city authorities in Europe and North America that are charged with the task of encouraging the revitalorisation and redevelopment of urban areas are now finding that $[\ldots]$ they lack the resources, power and control to implement formal masterplans. Instead some are beginning to experiment with looser planning visions and design frameworks, linked to phased packages of smaller, often temporary initiatives, designed to unlock the potential of sites now, rather than in 10 years' time. (Bishop et Lewis, 2012, p. 3)

C'est dans ce contexte, à partir des années 2010, que les milieux francophones commencent à parler d'urbanisme transitoire. L'Institut d'aménagement et d'urbanisme de la région Île-de-France distingue cette pratique de l'urbanisme éphémère ou tactique, affirmant que :

L'adjectif transitoire suggère, lui, que l'initiative s'inscrit dans une histoire connectée, pas seulement une juxtaposition d'usages sans lien avec l'avenir du territoire. Le projet d'urbanisme transitoire peut ainsi constituer un facteur de transition du lieu, de son image, de ses usages, et ainsi de son futur... participant aux grandes transitions vitales: énergétique, écologique et économique. (IAU, 2018a, p. 4)

En d'autres mots, l'urbanisme transitoire serait plus conscient du territoire d'insertion des initiatives temporaires et plus désireux de s'inscrire dans le temps. Plusieurs auteurs s'entendent d'ailleurs pour dire que l'urbanisme transitoire a la particularité d'avoir une visée pérenne (Adisson, 2017; Ginez, 2018; Plateau urbain, 2018). Pradel, pour qui l'urbanisme temporaire avait déjà la visée d'influencer le territoire de façon durable, parle plutôt d'un urbanisme à visée programmatique :

L'urbanisme transitoire, dont la vocation d'occupation temporaire de locaux vacants ou d'espaces ouverts par une diversité d'usages et d'usagers, est considéré comme une étape d'enrichissement et/ou de valorisation programmatique d'un projet d'aménagement à venir. (Pradel, 2018)

En d'autres mots, l'urbanisme transitoire serait une forme d'occupation temporaire organisée comme une étape dans la requalification pérenne d'un site. L'urbanisme transitoire semble ainsi vouloir contrôler l'impact négatif du temporaire sur les quartiers, sur les collectivités et sur leurs milieux de vie. 
D'une part, en planifiant la sortie des occupants temporaires, on limite leur précarisation. D'autre part, en participant au développement du projet pérenne, on favorise les liens entre occupants et propriétaires, et facilite l'intégration d'initiatives temporaires au marché permanent.

Bien que la définition de Pradel repose principalement sur les motivations de la pratique, l'auteur souligne également la diversité des usages et des usagers. Or, si l'on se réfère à l'Institut d'aménagement et d'urbanisme, il n'est pas tellement question ici d'une variété des projets d'urbanisme transitoire, mais plutôt de la mutualisation des espaces par une diversité d'acteurs (IAU, 2018a). Par exemple, entre 2015 et 2017, le projet des Grands Voisins, situé dans un ancien hôpital de la Saint-Vincent-de-Paul à Paris, a accueilli 250 associations, jeunes entreprises (start-ups), artisans et artistes. Dans ce contexte, ces initiatives sont d'ailleurs très souvent générées et opérées par des gestionnaires du transitoire, des collectifs « spécialisés dans l'occupation de lieux désaffectés de façon transitoire » (Adisson, 2017, p. 1). Ces collectifs sont encore assez différents les uns des autres, mais ils ont la particularité de travailler de pair avec les acteurs territoriaux (municipalités, communes ou arrondissements) et les propriétaires en amont de l'occupation.

$\mathrm{Si}$ ces initiatives sont planifiées et autorisées (Adisson, 2017), elles ne répondent toutefois pas, du moins pas officiellement, d'une volonté d'empêcher les occupations illégales, comme c'est le cas avec certaines initiatives ouvertement anti-squats. Il s'agirait plutôt pour les propriétaires de « tirer parti d'occupations temporaires légales» (Adisson, 2017, p. 1). Il n'est toutefois généralement pas question d'aider un propriétaire à tirer profit de son bâtiment vacant, mais plutôt de faire un pont entre des espaces vacants et des initiatives ou individus en mal d'espace à prix modique.

La gouvernance de ces projets est d'ailleurs tissée autour d'un trio d'acteurs principaux : la collectivité locale, les propriétaires et les occupants. Ainsi, il y a une mutualisation des espaces, un partage du poids financier relié à l'espace et aux responsabilités de gestion immobilière. L'urbanisme transitoire est alors perçu comme une manière d'engager les modes du temporaire à des fins durables et viables pour la collectivité. Cette dernière est d'ailleurs au cœur des initiatives transitoires, même si l'on remarque depuis quelques années en Europe l'arrivée de nouveaux acteurs gestionnaires qui ont une visée plus lucrative que sociale.

Cette pratique distincte, planifiée et autorisée, qui prône le réemploi d'espaces vacants à des fins collectives, semble à priori remarquable. Pourtant, si l'urbanisme transitoire se targue de limiter la gentrification en assurant la participation de la collectivité, il est toutefois encore difficile d'en mesurer les réels impacts dans un contexte où la pratique est nouvelle et où les processus que développent actuellement les différents gestionnaires transitoires n'ont pas encore été testés. Aussi, même si les villes semblent adhérer au mouvement, il reste beaucoup de travail à faire pour assurer une participation et une inclusion citoyennes qui reflètent les aspirations du transitoire.

\section{L'urbanisme transitoire à Montréal : accès au logement, développement culturel et préservation du patrimoine}

Les usages temporaires d'immeubles vacants font une apparition remarquée à Montréal à partir des années 1990. Organisé en 1997 par l'organisme Quartier éphémère, l'événement Panique au Faubourg a marqué l'imaginaire de plusieurs générations d'aménageurs et d'urbanistes montréalais. Depuis ce projet, des actions plus ou moins formelles se sont succédé, rassemblant une hétérogénéité d'acteurs porteurs d'une même volonté de combler le vide laissé par la vacance et par l'abandon de divers sites urbains.

En 2017, la promotion par la Ville de Montréal d'outils et de stratégies de valorisation des usages transitoires correspond à un autre moment charnière (Montréal transitoire, 2017). Que s'est-il passé entre 1997 et 2017? Quels sont les jalons du transitoire à Montréal?

Cette partie vise à défricher la façon dont l'urbanisme temporaire, en tant que mouvement social et culturel, s'est développé à Montréal et dans quel contexte nait l'urbanisme transitoire. Plus précisément, nous souhaitons mettre en évidence les expériences montréalaises qui alimentent les pratiques et les représentations des acteurs du transitoire. Pour ce faire, nous porterons attention aux 
initiatives phares d'occupations organisées de bâtiments vacants ou abandonnés à la lumière des éléments théoriques exposés dans la première partie.

\subsection{Le tournant du squat d'Overdale-Préfontaine : de la lutte pour le logement à l'animation socioculturelle}

Pour comprendre comment se constitue l'urbanisme transitoire à Montréal, il est nécessaire d'effectuer un rapide retour historique sur les cas recensés de squats. Si l'occupation d'un lieu vacant sans la permission de son propriétaire est une pratique courante en Europe (Van der Steen et collab., 2014), très peu de cas ont été recensés sur le territoire montréalais (Désy, 1993; Parazelli, Mensah et Colombo, 2010). Comme c'est le cas en Europe, ces pratiques apparaissent à Montréal au lendemain de la Seconde Guerre mondiale. À cette époque, la Ligue des vétérans sans-logis organise l'occupation illégale de bâtiments vacants pour loger environ 70 familles. L'événement sera désigné plus tard par Marc Choko (1980) comme le «mouvement de squatters de 1946-1947». Son action marque un précédent dans la dénonciation par des travailleurs de la pénurie de logements à Montréal.

En août 1975, le mouvement suit encore une fois les tendances européennes. Un groupe de citoyens impliquant des étudiants à faible revenu et des personnes âgées occupe environ 50 logements sur la rue SaintNorbert pour empêcher la démolition de bâtiments que souhaite l'administration Drapeau afin d'y aménager une cour de voirie. Comme le prédisaient les occupants incrédules, des condominiums luxueux y ont été édifiés, en dépit de l'avis des pouvoirs publics (Vallée, 2001).

De 1987 à 1989, une séquence semblable se produit à l'îlot Overdale: les squatteurs, des citoyens engagés dans la lutte pour le droit au logement, protestent contre la démolition contestée de logements jugés insalubres par l'administration Doré pour y construire des immeubles en copropriété (Favreau, 1988). Quelques années plus tard, en juillet 2001, la maison Louis-Hippolyte-Lafontaine de l'îlot Overdale, édifice classé bâtiment patrimonial, est à nouveau squattée. Pour la première fois, la dimension socioculturelle est clairement affichée dans les revendications des squatteurs. Comme le soulignent Parazelli et ses collaborateurs (2010), « outre la dénonciation de la pénurie de logements et la revendication de logements sociaux, l'objet de la négociation entre les squatters et le représentant de la Ville était un projet de coopérative d'habitation autonome appelé "Collectif Préfontaine" doté d'un complexe de diffusion culturelle alternative» (p. 156). Cependant, comme le déplore Mathieu Grondin, un des porteparoles des squatteurs, ce projet à visée culturelle ne sera pas développé : «On l'a perdu de vue, mais, au départ, le projet d'occupation était un gros projet culturel et mixte, avec une salle de spectacle et des logements pour les artistes » (Lecavalier, 2011, s. p.).

L'épisode d'Overdale-Préfontaine marque ainsi une forme de rattrapage des motivations des squats observés dans d'autres grandes villes européennes telles que Paris, Amsterdam ou Berlin. Pour reprendre les catégories d'analyse de Caroline Désy (1993), les squats les plus récents sont entrepris dans une visée culturelle, en comparaison des premiers squats d'urgence ou des squats plus politiques des décennies 1970 et 1980. Les premiers visaient la protection des citoyens dans une période de crise, alors que les seconds revendiquaient le droit à un logement, protestant contre le laissezfaire qui entourait les logements laissés vacants.

Deux cas récents de squat illustrent également ce tournant culturel. En 2009, l'occupation ratée de l'usine Seracon près du canal de Lachine débouche sur le projet Bâtiment 7, dont nous parlerons plus amplement plus loin. L'exemple du squat Fattal à SaintHenri est également pertinent. Ce bâtiment industriel abandonné depuis 25 ans a été transformé en studios d'artistes et, chaque année, un festival de musique punk y est organisé : le Fattal Fest. Le site de la Fattal a toutefois la particularité d'être assez exclusif, ouvert à une population bien spécifique et relativement fermé sur la collectivité locale. Il est intéressant de constater qu'en 2012, la Ville a tenté d'expulser les occupants en raison d'un zonage incorrect, mais l'intervention a échoué (Myers, 2015). Actuellement, les occupants des lieux paieraient un loyer modique au propriétaire (Daries, 2017).

Cette entente, très courante en Angleterre et aux PaysBas, permet aux squatteurs et aux propriétaires d'éviter les expulsions et les frais de poursuites judiciaires en signant des baux précaires ou des conventions d'occupation (Van der Steen et collab., 2014). L'amélioration de l'environnement local, la mise sur pied d'événements festifs, la programmation d'activités de loisirs ou d'éducation populaire ou encore la création 
de projets sociaux sont autant de leviers expérimentaux de participation culturelle au sein des squats les plus organisés. Dans ces cas, on alimente et justifie des démarches légales, mais informelles d'occupation de bâtiments inusités. Cette porosité entre l'illégalité et la légalité est particulièrement visible lors des phases préliminaires de renouvellement urbain du Faubourg des Récollets, où les concerts underyround ont rencontré les premières interventions artistiques des sites industriels abandonnés.

\subsection{Quartier éphémère au Faubourg des Récollets : la stratégie de la visibilité médiatique}

Au début des années 1990, préoccupée par la dégradation de son patrimoine industriel, la Ville de Montréal propose à l'association française Usines éphémères, dont la mission est de requalifier les friches urbaines en espaces de création, d'investir les sites vacants près du canal de Lachine. En 1993, l'association crée Quartier éphémère, une organisation sans but lucratif dédiée à la revalorisation et à la réhabilitation de l'héritage industriel montréalais, assurant ainsi « le passage entre deux histoires, deux possibles d'un bâtiment » (Point éphémère, 2011, s. p.).

Dès 1997, Quartier éphémère met en œuvre le projet Panique au Faubourg. Dix artistes installent leurs œuvres temporaires dans plusieurs bâtiments vacants situés dans un ancien quartier industriel en déclin : le Faubourg des Récollets. En juin 1998, le gouvernement du Québec, en collaboration avec la Ville de Montréal, lance toutefois la Cité du Multimédia dans ce même quartier. Le projet urbain vise à regrouper les entreprises en technologies de l'information et de la communication grâce à des mesures d'aide fiscale.

Selon Claire Poitras (2002), la reconversion culturelle des bâtiments industriels a ainsi participé à la création d'une image de marque prisée par les promoteurs immobiliers, qui ont ensuite soutenu les grandes opérations de transformation du quartier. Ces promoteurs ont justifié leur action en s'appuyant sur les impacts jugés néfastes de la désindustrialisation: des structures désuètes, des sites contaminés et plein de dangers potentiels (Poitras, 2002). Or, si l'initiative Panique au Faubourg a été un succès public, les interventions artistiques n'ont toutefois pas réussi à charger suffisamment de sens et de valeur symbolique aux édifices pour mener vers une préservation des spécificités architecturales et morphologiques des lieux.
Au contraire, les opérations de rénovation ont fortement atténué les caractéristiques architecturales des anciens bâtiments industriels pour correspondre à leur nouvelle vocation (Bélanger, 2011). Cet épisode montre toutes les limites d'une stratégie de visibilité médiatique basée sur la valorisation et la préservation de sites délaissés. À cet égard, le cas de la Fonderie des frères Darling est un meilleur exemple de reconversion industrielle réussie. Le tout grâce à une solide mobilisation citoyenne et à l'implication continue d'occupants engagés.

\subsection{Atelier In situ et la Fonderie Darling : la stratégie de la convivialité à l'extérieur des murs}

Parmi les œuvres de Panique du Faubourg, celle de Claude Lévesque a contribué à porter plus spécifiquement l'attention sur l'ancienne Fonderie des frères Darling, reconvertie en un centre d'arts visuels. Dès 1995, l'idée d'un espace de création artistique est soumise par La Société du 5 avril, mais le projet n'aboutit pas. L'œuvre de Lévesque fut alors le déclencheur d'un long processus de transformation du bâtiment à l'abandon. La signature d'un bail de très longue durée entre la Société de développement de Montréal (SDM) et l'organisation Quartier éphémère, puis l'acquisition du bâtiment en 2004 ont permis la pérennité du projet dédié à la création, à la production et à la diffusion artistiques.

Comme le rapporte Mardjane Amin (2015), la réhabilitation du site s'effectue en deux phases. La première est menée entre 2001 et 2002 par Atelier In situ. Cette jeune agence d'architectes effectue une multitude d'interventions légères sur le bâtiment pour réhabiliter minimalement le lieu et pour permettre une occupation confortable. De 2003 à 2006, la seconde phase est prise en charge par le cabinet d'architectes L'CEUF, spécialisé dans les constructions durables, afin d'aménager les studios d'artistes, tout en prenant soin de conserver la mémoire industrielle du lieu. Depuis 2007, les occupants de la Fonderie Darling investissent ponctuellement le tronçon de la rue Ottawa entre les rues Queen et King. Plusieurs installations temporaires telles que des tables de piquenique, des jardins communautaires, un skatepark éphémère, des événements musicaux, etc. sont mis en œuvre sur cette "place publique» pour animer le voisinage de proximité et pour créer des espaces de convivialité tout au long de l'année. 
La stratégie de reconversion de la fonderie doit ainsi être comprise dans ce rapport à son quartier d'insertion. Dans une enquête sur ce cas de reconversion, Shauna Janssen (2009) rapporte les propos de la fondatrice de la Fonderie Darling, qui expliquait cette stratégie visant à créer une proximité avec le milieu pour s'en faire un allié politique et pour agir durablement sur le devenir du quartier: «For me, the physical presence of the artist in the community is very important too. Artists are like philosophers. They make links in between. They create communities » (p. 90).

Même si les acteurs de ce projet ne parlent pas encore d'urbanisme transitoire, la stratégie d'occupation utilisée ici s'en rapproche : l'urbanisme tactique est utilisé en amont d'une occupation pérenne, autorisée et planifiée, dans le but de préparer l'arrivée du projet et d'assurer le soutien de la collectivité locale. En ce sens, le cas de la Fonderie Darling montre également que l'urbanisme transitoire dépasse les seules dimensions d'occupation et de remise à niveau d'un bâtiment vacant. C'est aussi l'expression d'un rapport social, culturel et politique entre une diversité d'acteurs qui se projettent sur le devenir d'un bâtiment, construisent des solidarités et prennent en main le développement d'un quartier.

\section{$2.4 \quad$ Le Collectif 7 à nous et le Bâtiment 7 : de la stratégie du fait accompli à la combinaison des moyens de pression}

Au début des années 2000, un foyer de militants radicaux se constitue à Pointe-Saint-Charles pour lutter contre les effets des transformations urbaines que subissent le quartier et les classes populaires depuis la réouverture du canal de Lachine en 2002. C'est dans ce contexte qu'émergent deux organisations militantes créées en 2004 et 2007 : la Pointe Libertaire et le Centre social autogéré (CSA). Porteurs de valeurs anarchistes, ces collectifs mènent plusieurs actions communautaires, dont des ateliers de réparation de vélos, des projections de films, des concerts, des cours d'éducation populaire, des repas collectifs, etc. En 2007 , ils annoncent leur volonté de rassembler ces activités au sein d'un lieu pérenne.

En mai 2009, une première action directe de squatting est organisée à l'usine Seracon, qui doit être convertie en condominiums. Cette action mobilise 70 organisations signataires d'une déclaration de solidarité et
500 manifestants. En une journée, les squatteurs installent une cuisine, des toilettes, des dortoirs et une scène. Cette stratégie du fait accompli échoue et les militants sont expulsés le jour suivant.

Le Bâtiment 7, un édifice de 90000 pi² localisé sur d'anciens terrains du Canadien National $(\mathrm{CN})$, devient alors la cible prioritaire du regroupement (Kruzynski et Silvestro, 2013). Le bâtiment, promis à la démolition, avait déjà été l'objet de plusieurs tentatives de reconversion. Avant la fermeture définitive du site par Alstom, la Fonderie Darling y avait d'ailleurs ouvert quatre ateliers d'artistes en 2003. Deux opérations populaires d'aménagement ont ensuite été mises en œuvre en 2004 et 2007 pour influencer l'aménagement futur du site. Enfin, deux autres exercices de consultation sur la reconversion des terrains vacants du CN ont été menés par l'Office de consultation publique de Montréal (OCPM) en 2008 et 2009.

Au printemps 2009, le Collectif 7 à nous, composé d'une multitude hétéroclite d'anarchistes, de résidents et d'acteurs communautaires, est créé. Il réclame que le Bâtiment 7 soit cédé gratuitement à la communauté pour accueillir des projets socioculturels. Après plusieurs années de luttes et d'actions tactiques, un accord est signé en 2011 entre le propriétaire et les représentants du Collectif 7 à nous. L'entente inclut la cession du Bâtiment 7, la décontamination du terrain et un don de 1 million de dollars pour la rénovation du bâtiment. Or, si l'accord est entériné par l'arrondissement du Sud-Ouest, la Ville de Montréal bloque le dossier, avant d'accepter l'accord en octobre 2012 (Perron, 2012). L'accord devient en vigueur le 28 avril 2017 et l'ouverture se déroule l'année suivante.

Aujourd'hui, le Bâtiment 7 est un lieu de rassemblement qui accueille quatre entreprises collectives (une épicerie autogérée, une école d'art et deux coopératives), des ateliers de fabrication collaboratifs (mécanique, sérigraphie, bois, impression numérique, céramique, photographie) et des espaces communs (salle polyvalente, salle de réunion, douches, cuisine, rangement) administrés par le Collectif 7 à nous. La mobilisation citoyenne aura toutefois duré plus d'une décennie pour arriver à la reconversion de ce bâtiment industriel. Perron (2012) et Lachapelle (2017) ont pu constater que la cohabitation et la collaboration entre des acteurs hétérogènes se sont avérées être un atout pour la lutte en faveur de l'appropriation du bâtiment: 
Pendant que l'influente Fonderie Darling négociait directement avec V. Chiara, la table de concertation Action-Gardien - composée d'une trentaine de groupes communautaires locaux - talonnait les élus locaux et mobilisait les résidents du quartier. Le CSA, plus contestataire et propulsé par l'action directe, agissait comme si le bâtiment était déjà «à nous » en organisant des squats ponctuels, festifs et familiaux. (Kruzynski, 2017, p. 143)

Ces acteurs ont su allier plusieurs formes de stratégies complémentaires : la confrontation, l'alliance avec le milieu et la négociation.

\subsection{Entremise et le projet Young : l'intermédiation au service de l'innovation sociale}

En 2016, l'équipe Entremise gagne le concours d'idée Morph.o.polis de l'Office de consultation publique de Montréal. Défendant l'idée que la vacance urbaine est une occasion de développer, elle a pour mission de " connecter des espaces sans personnes à des personnes sans espaces ». Cet organisme à but non lucratif se rattache d'emblée à l'urbanisme transitoire. Rapidement, la Ville de Montréal, par l'intermédiaire de la Division du patrimoine, est un interlocuteur privilégié. Au printemps 2016, la Ville organise les Entretiens sur le patrimoine, qui sont précurseurs du Plan d'action en patrimoine, adopté un an plus tard.

C'est dans ce contexte qu'on invite Entremise à participer aux discussions, dans le but d'élargir les débats sur la gestion du patrimoine. C'est à ce moment qu'émerge, sur le plan politique, l'idée des usages transitoires à Montréal. La Ville de Montréal et Entremise organisent ensuite l'événement Montréal transitoire, dans le but de réunir des acteurs diversifiés afin de démystifier la pratique, et de réfléchir au potentiel et aux difficultés qui entourent la mise en place de ces pratiques dans la métropole.

Deux éléments ressortent des discussions : la volonté d'agir en mettant en œuvre des projets pilotes et l'engagement de la Ville en tant que propriétaire immobilier pour assurer la mise en place d'une règlementation adéquate. Néanmoins, aucun accord n'émerge sur la manière de faire les usages transitoires au sein des différents services de la ville-centre, et la structure institutionnelle qui accompagne les questions d'urbanisme est lourde, rigide et peu propice aux initiatives qui sortent des sentiers battus (Baillargeon, 2018). On est alors en pleine période électorale et l'arrivée de l'équipe Projet Montréal à la mairie participe à faire débloquer le processus.

Dans la programmation proposée par Projet Montréal (2017), l'équipe encourage les usages transitoires de bâtiments vacants et propose de « créer une "division de l'occupation transitoire" pour prendre en charge les terrains et les bâtiments inoccupés et réduire le taux de vacance des locaux disponibles » (p. 23). Projet Montréal est élu le 5 novembre 2017 et, quatre mois plus tard, le premier projet d'urbanisme transitoire ouvre ses portes dans un ancien hangar de la Ville de Montréal. La promesse est ainsi tenue. Non seulement un premier projet est lancé, mais la Ville se fait leader exemplaire en la matière. Dans cette configuration, Entremise développe une première initiative concrète, en collaboration avec la Maison d'innovation sociale, la Fondation McConnell et la Division du patrimoine : le Laboratoire transitoire.

L'idée de départ de ce projet est d'ouvrir une série de projets pilotes réalisés en partenariat avec la Ville de Montréal, l'initiative Cities for People de la Fondation McConnell et la Maison de l'innovation sociale. Le projet Young constitue la première étape de ce projet laboratoire. Le hangar, situé au 204, rue Young, à Griffintown, est choisi pour accueillir ce projet. Cet espace ouvert de 5000 pi² est sous-occupé depuis déjà plusieurs années et on y prévoit la construction d'un projet de logements en 2020.

Lorsque l'OBNL lance son appel à candidatures pour l'occupation de cet espace en décembre 2017, les critères de sélection sont plutôt larges : il faut avoir besoin d'un espace abordable, avoir de potentielles retombées sur la communauté locale, faire preuve d'un désir de vie commune et avoir une synergie entre les différentes initiatives choisies. Entremise reçoit 53 candidatures et 17 projets sont choisis. Entremise entreprend parallèlement une série de travaux minimes pour rendre le lieu usuel et accueillant. Ce sont les occupants qui aménagent ensemble l'essentiel de l'espace. En effet, chaque occupant est chargé d'aménager son propre espace de bureau, tandis que les espaces communs (cuisine, salle polyvalente et salle de réunion) sont coaménagés. Le projet, entamé en février 2018, s'est terminé le 31 décembre 2019. 
Un an et demi après l'ouverture du projet Young, il n'y a toutefois toujours pas d'autres projets d'urbanisme transitoire instauré par le partenariat du Laboratoire transitoire. Force est d'admettre que, malgré la présence de l'urbanisme transitoire dans les discours de la ville-centre, cette pratique n'est pas aussi simple à mettre en place qu'elle n'y parait. En effet, ce projet a nécessité plusieurs détournements au sein de la municipalité. Qui plus est, la gestion de l'espace n'est pas une mince affaire et les frais de fonctionnement, bien qu'assez bas, restent lourds pour un organisme encore peu expérimenté. Aussi, à l'échelle de la ville-centre, plusieurs changements sont effectués dans la gestion des services internes, ce qui ne sera pas sans complexifier le nouveau projet. Une structure municipale en silo et une grande difficulté de fonctionnement entre les divers services concernés par le transitoire semblent expliquer l'essentiel du ralentissement de leur part.

Néanmoins, en l'espace de quatre ans, la stratégie de collaboration étroite d'Entremise avec des acteurs clés a permis un changement de posture de milieu sur les enjeux des usages transitoires en faisant accepter une idée novatrice au sein d'une administration publique. Si le projet Young marque un précédent dans la pratique urbanistique à Montréal du fait qu'il expérimente selon d'autres logiques la reconversion de bâtiments vacants ou abandonnés, on peut toutefois regretter le faible impact du projet au sein de son quartier de proximité. Un défaut que les autres projets transitoires à venir devront pallier.

\section{Conclusion}

\section{Le transitoire : un projet de société?}

Alors qu'en Europe, l'urbanisme transitoire est déjà bien développé, il n'existe encore que très peu de projets du genre à Montréal. Historiquement, la reconversion des bâtiments vacants ou abandonnés est principalement portée par la société civile, par le milieu communautaire ou par des acteurs sensibles à la conservation du patrimoine. Ce premier état des lieux de l'urbanisme transitoire montréalais indique une diversité de stratégies d'action allant de la confrontation vers la collaboration, en passant par la négociation, l'alliance avec le milieu d'insertion ou la médiatisation artistique. L'annonce par la Ville de Montréal de lancer la reconversion de 10 bâtiments en espaces partagés ouverts sur la communauté montre l'émergence d'un processus d'institutionnalisation d'un mode d'intervention pour résoudre le problème de la vacance et de l'abandon. À l'image d'Entremise, la professionnalisation des organismes engagés dans la reconversion des bâtiments est en cours pour faire face à la complexité des projets, à la diversité des acteurs impliqués et aux obstacles règlementaires rencontrés par les porteurs de projets transitoires.

L'augmentation de la compétence et de la qualité d'exécution apportera nécessairement son lot de contradictions et de contraintes. En effet, la professionnalisation de ces pratiques peut encourager des évolutions décisives en matière de règlementation, d'aménagement ou de politiques publiques pour favoriser la réutilisation de bâtiments inusités. Ces innovations pourront alors être adaptées et améliorées dans d'autres contextes urbains.

Néanmoins, à l'instar du mouvement de l'urbanisme tactique, la professionnalisation laisse présager une forme d'uniformisation des interventions, voire de labellisation des projets entrepris par les groupes les plus organisés et, à terme, par les pouvoirs publics eux-mêmes. Or, comme l'écrit l'urbaniste Patrick Bouchain (2006) : «Ce n'est pas parce qu'un ouvrage est bien fait qu'il est chargé de sens » (p. 94). Les moments de confrontation, puis de négociation vécus durant la mise en place de la Fonderie Darling et du Bâtiment 7 ont permis de créer un sentiment d'appartenance autour des projets. L'effacement de ces formes de résistance issues de la culture du squat ne risque-t-il pas alors de ralentir les processus d'appropriation citoyenne des lieux ainsi que l'ancrage territorial des projets?

Cet article visait également à identifier de nouvelles pistes de recherche sur le cas montréalais. Il serait intéressant d'affiner notre compréhension des valeurs, des engagements, des motivations, des parcours et des logiques d'actions des acteurs engagés dans l'urbanisme transitoire. Ces projets sont mobilisés en faveur d'un public et d'un territoire en reposant sur une diversité d'offres et d'activités. Quelles sont les retombées de ces projets sur leur milieu d'insertion? Quels sont les impacts positifs et négatifs? Pour qui? La constitution de ces savoirs permettra d'identifier les atouts et les faiblesses de l'urbanisme transitoire à Montréal vis-à-vis d'autres métropoles. 


\section{RÉFÉRENCES}

Adisson, F. (2017). Choisir ses occupants : quand les grands propriétaires adoptent des collectifs pour la gestion transitoire des friches urbaines. Métropolitiques. Repéré à www.metropolitiques.eu/Choisir-ses-occupants.html

Ambrosino, C. et Andres, L. (2008). Friches en ville : du temps de veille aux politiques de l'espace. Espaces et sociétés, 134, 37-51. https://doi.org/10.3917/esp.134.0037

Amin, M. (2015). De l'édifice industriel à l'espace artistique : quelle place pour la conservation du patrimoine industriel et technique? (Mémoire de maitrise non publié). Université de Montréal, Montréal.

Andres, L. (2011). Alternative initiatives, cultural intermediaries and urban regeneration: The case of La Friche (Marseille). European Planning Studies, 19(5), 795-811. https://doi.org/10.1080/09654313.2011.561037

Andres, L. (2013). Differential spaces, power hierarchy and collaborative planning: A critique of the role of temporary uses in shaping and making places. Urban Studies, 50, 759-775. https://doi.org/10.1177/0042098012455719

Baillargeon, T. (2018). Montréal transitoire : une nouvelle façon de penser et d'habiter la ville. Urbanité, automne, 34-36. Repéré à https://ouq.qc.ca/wp-content/uploads/2018/10/urbanite-automne2018-web.pdf

Beaudet, G. (1997). Domaines « vides » et structuration morphologique de l'agglomération montréalaise. Cabiers de géographie du Québec, 41(112), 7-29. https://doi.org/10.7202/022606ar

Beekmans, J. et De Boers, J. (2014). Pop-up city: City-making in a fluid world. Amsterdam, NL: BIS Publisher.

Bélanger, M. (2011). Vestiges industriels montréalais : appropriations, rôles et enjeux sociaux (Mémoire de maitrise). Université de Montréal, Montréal. Repéré à https://papyrus.bib.umontreal.ca/xmlui/handle/1866/5498

Bishop, P. et Williams, L. (2012). The temporary city. Oxford, R.-U.: Routledge.

Bossetti, N. et Colthorpe, T. (2018). Meanwhile, in London: Making use of London's empty spaces. Londres, R.-U. : Centre for London. Repéré à www.centreforlondon.org/reader/meanwhile-use-london

Bouchain, P. (2006). Construire autrement. Paris, France : Actes Sud.

Colomb, C. (2012). Pushing the urban frontier: Temporary uses of space, city marketing, and the creative city discourse in 2000s Berlin. Journal of Urban Affairs, 34(2), 131-152. https://doi.org/10.1111/j.1467-9906.2012.00607.x

Chatterton, P. et Hollands, R. (2003). Urban nightscapes: Youth cultures, pleasure spaces and corporate power. Londres, R.-U.: Routledge.

Chaudoir, P. (2007), La ville événementielle : temps de l'éphémère et espace festif. Géocarrefour, 3, 107-110. Repéré à http://journals.openedition.org/geocarrefour/2301

Choko, M. (1980). Crise du logement à Montréal (1860-1939). Montréal, QC : Éditions coopératives Saint-Martin.

Daries, M. (2017, 15 février). Les squats à Montréal : inégalités sociales et parcours de vie. [Billet de blogue]. Repéré à http://inegalitessociales.com/2017/02/15/les-squats-a-montreal

Désy, C. (1993). S’approprier un coin du monde et s'enraciner: les squatters. Nowvelles pratiques sociales, 6(1), 143-154. https://doi.org/10.7202/301204ar

Doron, G. M. (2007). ...badlands, blank space, border vacuums, brown fields, conceptual Nevada, Dead Zones, derelict areas, ellipsis spaces, empty places, free space liminal spaces, nameless spaces, No Man's Lands, polite spaces, post architectural zones, spaces of indeterminacy, spaces of uncertainty, smooth spaces, Tabula Rasa, Temporary Autonomous Zones, terrain vague, urban deserts, vacant lands, voids, white areas, Wasteland... SLOAPs. Field Journal, 1(1), 10-23. Repéré à www.field-journal.org/ uploads/file/2007_Volume_1/g\%20doron.pdf

Douglas, G. C. C. (2013). Do-it-yourself urban design: The social practice of informal “improvement” through unauthorized alteration. City and Community, 13(1), 5-25. https://doi.org/10.1111/cico.12029

Dubeaux, S. (2017). Les utilisations intermédiaires des espaces vacants dans les villes en décroissance : transferts et transférabilité entre l'Allemagne et la France (Thèse de doctorat). Université Paris Sciences et Lettres, Paris.

Repéré à www.aurh.fr/media/dubeaux2017_these_vf_adum_065854500_1552_08032018.pdf

Favreau, M. (1988, 28 juin). Les résistants d’Overdale débusqués : la police force l'évacuation de 2 logis jugés dangereux 13 arrestations. La Presse, p. 3. 
Garvin, E., Branas, C., Keddem, S., Sellman, J. et C. Cannuscio. (2013). More than just an eyesore: Local insights and solutions on vacant land and urban health. Journal of Urban Health: Bulletin of the New York. Academy of Medicine, 90(3), 412-426. https://doi.org/10.1007/s11524-012-9782-7

Ginez, C. (2018). Définir l'urbanisme temporaire au sein du projet urbain : un outil institutionnel innovant? Au prisme des collectivités locales: aménagement transitoire du square Augereau à Albi (81) (Mémoire de Master 2). Université Grenoble Alpes, Grenoble. Repéré à https://dumas.ccsd.cnrs.fr/dumas-01870163/document

Groth, J. et Corijn, E. (2005). Reclaiming urbanity : Indeterminate spaces, informal actors and urban agenda setting. Urban Studies, 42(3), 503-526. https://doi.org/10.1080/00420980500035436

Harvey, D. (2012). Rebel cities. Brooklyn, NY: Verso.

Haydn, F. et Temel, R. (2006). Temporary urban spaces: Concepts for the use of the city spaces. Bâle, Suisse: Birkhauser.

Hou, J. (dir.). (2010). Insurgent public space: Guerrilla urbanism and the remaking of contemporary cities. Londres/New York : Routledge.

Institut d'aménagement et d'urbanisme (IAU) de la région Île-de-France. (2018a). L'urbanisme transitoire : optimisation foncière ou fabrique urbaine partagée? Paris : IAU Île-de-France. Repéré à www.institutparisregion.fr/fileadmin/NewEtudes/Etude_1427/UrbanismeTransitoire.pdf

Institut d'aménagement et d'urbanisme (IAU) de la région Île-de-France. (2018b). Urbanisme transitoire : une nouvelle manière de fabriquer la ville? Paris : IAU Île-de-France. Repéré à www.institutparisregion.fr/fileadmin/DataStorage/Recherche/ PetitDej/2018/ENS_Urbanisme_transitoire.pdf

Iveson, K. (2013). Cities within the city: Do-it-yourself urbanism and the right to the city. International Journal of Urban and Regional Research, 37(3), 941-956. https://doi.org/10.1111/1468-2427.12053

Janssen, S. (2009). Reclaiming the Darling Foundry from post-industrial landscape to Quartier Ephemere, (Mémoire de maitrise). Université Concordia, Montréal. Repéré à https://spectrum.library.concordia.ca/976466

Jégou, F. et Bonneau, M. (2016). REFILL: Reuse of vacant spaces as driving force for innovation on local level. Urbact. Repéré à https://urbact.eu/sites/default/files/refill_160515_soa_final_to_be_published_0.pdf

Korosec-Serfaty, P. (1991). La ville et ses restes. Dans A. Germain (dir.), L'aménagement urbain : promesses et défis (p. 233-267). Montréal, QC : Institut québécois de recherche sur la culture.

Kruzynski, A. (2017). L'autonomie collective en action : du Centre social autogéré de Pointe-Saint-Charles au Bâtiment 7. Nowvelles pratiques sociales, 29(1-2), 139-158. https://doi.org/10.7202/1043397ar

Kruzynski, A. et Silvestro, M. (2013). Proximité physique, vie de quartier et luttes anarchistes. Dans R. Bellemare-Caron, É. Breton, M.-A. Cyr, F. Dupuis-Déri et A. Kruzynski (dir.), Nous sommes ingouvernables : les anarchistes au Québec (p. 137-151). Montréal, QC : Lux.

Lachapelle, M. (2017, 30 mars). Espace d'autonomie et structures de contraintes : la mise en cewre du projet Bâtiment 7 à Montréal. Communication présentée au Colloque GRIR à l'UQAC, Chicoutimi, Québec.

Lecavalier, C. (2011, 11 août). Le squat Préfontaine. Le Journal de Montréal. Repéré à www.journaldemontreal.com/2011/08/11/ le-squat-prefontaine

Lefebvre, H. (1968). Le droit à la ville. Paris, France : Anthropos.

Lefebvre, H. (1970). La révolution urbaine. Paris, France : Gallimard.

Lefebvre, H. (1974). La production de l'espace. Paris, France : Anthropos.

Lévesque, L. (1999). Montréal, l'informe urbanité des terrains vagues : pour une gestion créatrice du mobilier urbain. Les Annales de la recherche urbaine, 85, 47-57. Repéré à www.persee.fr/doc/aru_0180-930x_1999_num_85_1_2280

Lydon, M. et Garcia, A. (2015). Tactical urbanism: Short-term action for long-term plan. Washington, DC: Island Press.

Mallach, A. (2006). Bringing buildings back: From abandoned properties to community assets. New Brunswick, NJ : Rutgers University Press.

Montréal transitoire. (2017). Réflexion collective sur les usages temporaires dans les bâtiments vacants : rapport d'événement. Repéré à http://ville.montreal.qc.ca/pls/portal/docs/page/proj_urbains_fr/media/documents/rapport_mtltransitoire_vf_2017.pdf

Mould, O. (2014). Tactical urbanism: The new vernacular of the creative city. Geography Compass, 8(8), 529-539. https://doi.org/10.1111/gec3.12146 
Myers, M. (2015, 1 septembre). Montreal's Fattal Fest is kinda like if "Mad Max" and "Waterworld" had a punk baby. Vice. Repéré à www.vice.com/en_ca/article/6w8emy/montreal-punk-fattal-fest-2015

Nefs, M. (2006). Unused urban space: Conservation or transformation? Polemics about the future of urban wastelands and abandoned buildings. City \& Time, 2(1), 47-58. Repéré à https://pdfs.semanticscholar.org/c61f/ e0ffd70a112668fd58f0138ff1ee5ec6a556.pdf?_ga=2.105180355.1960690748.1579878975-761685907.1579878975

Oswalt, P. (2005). Shrinking cities. Stuttgart, Allemagne : Hatje Cantz.

Oswalt, P., Overmeyer, K. et Misselwitz, P. (2013). Urban catalyst: The power of temporary use. Berlin, Allemagne: DOM Publishers.

Overmeyer, K. (dir.). (2007). Urban pioneers: Temporary use and urban development in Berlin. Berlin, Allemagne : Jovis.

Parazelli, M., Mensah, M. N. et Colombo, A. (2010). Exercer le droit au logement : le cas d'un épisode de squattage à Montréal en 2001. Lien social et Politiques, 63, 155-168. https://doi.org/10.7202/044157ar

Perron, R. (2012). Militantisme libertaire et action communautaire dans le quartier Pointe-Saint-Charles à Montréal : le cas du Collectif7 à nous (2009-2012) (Mémoire de maitrise). Université du Québec à Montréal, Montréal.

Repéré à https://archipel.uqam.ca/9391/1/M14628.pdf

Pinard, J. et Vivant, E. (2017). La mise en évènement de l'occupation temporaire : quand les lieux artistiques off inspirent les opérateurs de la production urbaine. L'Observatoire, 50, 29-32. https://doi.org/10.3917/lobs.050.0029

Plateau urbain. (2018). Urbanisme temporaire : définitions, acteurs, enjeux. Ekopolis. Repéré à www.ekopolis.fr/ressource/urbanismetemporaire-definitions-acteurs-outils-et-enjeux

Point éphémère (2011). [Sans titre]. Repéré à www.pointephemere.org/spip.php?article10

Poitras, C. (2002). La Cité du multimédia à Montréal : fabriquer l'image d'un nouveau quartier. Dans J. Malézieux, C. Manzagol et G. Sénécal (dir.), Grands projets urbains et requalification (p. 137-151). Sainte-Foy, QC : Presses de l’Université du Québec.

Pradel, B. (2018). Urbanisme temporaire, une définition? Kaléido'Scop. Repéré à www.kaleido-scop.com/Urbanisme-temporaire-une

Projet Montréal. (2017). Programme 2017. Repéré à https://d3n8a8pro7vhmx.cloudfront.net/projetmontreal/pages/1985/ attachments/original/1506441373/Programme_final.pdf?1506441373

Pruijt, H. (2003). Is the institutionalization of urban movements inevitable? A comparison of the opportunities for sustained squatting in New York City and Amsterdam. International Journal of Urban and Regional Research, 27(1), 133-157. https://doi.org/10.1111/1468-2427.00436

Schachterle, S. E., Bishai, D., Shields, W., Stepnitz, R. et Gielen, A. C. (2012). Proximity to vacant buildings is associated with increased fire risk in Baltimore, Maryland, homes. Injury Prevention, 18(2), 98-102. http://dx.doi.org/10.1136/injuryprev-2011-040022

Talen, E. (2015). Do-it-yourself urbanism: A history. Journal of Planning History, 14(2), 135-148. https://doi.org/10.1177/1538513214549325

Tonkiss, F. (2013). Austerity urbanism and the makeshift city. City, 17(3), 312-324. https://doi.org/10.1080/13604813.2013.795332

Urban Unlimited (2004). The shadow city. Repéré à https://issuu.com/urbanunlimited/docs/shadowcity

Vallée, B. (2001, 2 août). Histoire populaire : le squat d’Overdale n’était pas le premier au Québec, La Presse. Repéré à http://archives-2001-2012.cmaq.net/fr/node/6509.html

Van der Steen, B., Katzeff, A. et Van Hoogenhuijze, L. (2014). The city is ours: Squatting and autonomous movements in Europe from the 1970s to the present. Oakland, CA: PM Press.

Vasudevan, A. (2017). The autonomous city: A bistory of urban squatting. Londres, R.-U.: Verso.

Veschambre, V. (2008). Traces et mémoires urbaines : enjeux sociaux de la patrimonialisation et de la démolition. Rennes, France : Presses universitaires de Rennes.

Vidler, A. (1992). The arcbitecture uncanny. Boston, MA: MIT Press.

Zielh, M., Oßwald, S., Hasemann, O. et Schnier, D. (2012). Second hand spaces: Recycling sites undergoing urban transformation. Berlin, Allemagne : Jovis. 